\title{
Psychophysical Neuroeconomics of Decision Making: Nonlinear Time Perception Commonly Explains Anomalies in Temporal and Probability Discounting ${ }^{*}$
}

\author{
Taiki Takahashi", Ruokang Han \\ Center for Experimental Research in Social Science, Department of Behavioral Science, \\ Faculty of Letters, Hokkaido University, Sapporo, Japan \\ Email: "taikitakahashi@gmail.com
}

Received August 27, 2013; revised September 27, 2013; accepted October 5, 2013

Copyright (C) 2013 Taiki Takahashi, Ruokang Han. This is an open access article distributed under the Creative Commons Attribution License, which permits unrestricted use, distribution, and reproduction in any medium, provided the original work is properly cited.

\begin{abstract}
Anomalies in decision over time (e.g., "hyperbolic time discounting") and under risk (e.g., Allais paradox and hyperbolic probability discounting) have been attracting attention in behavioral and neuroeconomics. We have proposed that psychophysical time commonly explains anomalies in both decisions (Takahashi, 2011, Physica A; Takahashi et al., 2012, J Behav Econ \& Finance). By adopting the $q$-exponential time and probability discounting models, our psychophysical and behavioral economic experiment confirmed that nonlinear distortion of psychophysical time is a common cause of the anomalies in decision both over time and under risk (i.e., intertemporal choice and decision under risk). Implications for psychophysical neuroeconomics and econophysics are discussed.
\end{abstract}

Keywords: Psychophysics; Discounting; Neuroeconomics; Econophysics; Tsallis’ Statistics

\section{Introduction}

\subsection{Hyperbolic Discounting of Delayed and Probabilistic Rewards}

The relationship between decision over time and under risk has been of question in many areas such as decision theory, neuroeconomics and neuroscience [1-3]. Time discounting refers to the behavioral tendency of devaluating delayed rewards. Abundant empirical studies documented that people temporally discount rewards in a hyperbolic manner [4]:

$$
V(t)=V(0) /(1+k t)
$$

where $k$ is the degree of impulsivity at $t=0$.

According to Rachlin and colleagues (1991), time discounting underlies people's behavioral tendency to gamble whereby the probability $(p)$ of wining repeated gambles can be converted to odds against $(O)=1 / p-1$, which is the average number of trials until a win. The

\footnotetext{
${ }^{*}$ The research reported in this paper was supported by a grant from the Grant-in-Aid for Scientific Research (Adolescent Mind \& Self-Regulation) from the Ministry of Education, Culture, Sports, Science and Technology of Japan.

${ }^{\#}$ Corresponding author.
}

probability discounting was found to be in a hyperbolic form in terms of "odds-against" which corresponds to waiting time in repeated gambles [4]. Hence the hyperbolic probability discounting function is

$$
V(O)=V(0) /(1+k O)
$$

where $k$ is the degree to which the uncertain reward is discounted.

\subsection{Time Inconsistency and Psychophysical Time}

The normative economic theory assumes time consistency, which is described by an exponential time discount function [5]:

$$
V(t)=V(0) \exp (-k t)
$$

where time discount rate is constant (i.e.

$\left.\mathrm{DR}=-V(t)^{\prime} / V(t)=k\right)$. In contrast, the hyperbolic time discounting leads to time inconsistent choice (i.e. decreasing impatience), which violates the normative model [6].

In order to solve time inconsistency, Takahashi (2005) [7] has introduced nonlinear psychophysical time into the time discount function. According to Weber-Fechner law, 
psychophysical time function evaluated by physical time is logarithmic:

$$
\tau(t)=\alpha \ln (1+\beta t)
$$

where $\alpha$ and $\beta$ are free parameters. Thus the time discount model with logarithmic psychophysical time is as follows:

$$
V(t)=V(0) \cdot D(\tau(t))=\frac{V(0)}{(1+\beta t)^{k \alpha}}
$$

where $k$ is the degree of discounting in exponential discounting in terms of psychophysical time $\tau$. Therefore, introducing logarithmic psychophysical time (Equation (4)) into the time discount function (Equation (2)) can express a hyperbola-like function (Equation (5)). Han and Takahashi (2012) [8] empirically demonstrated that when logarithmic psychophysical time was introduced into the time discounting model, the functional form of time discount model was closer to exponential than hyperbolic, supporting Takahashi's logarithmic time perception theory of non-exponential discounting [7,9].

\subsection{Decision under Risk and Psychophysical Time}

In game theory, von Neumann and Morgenstern (1947) [10] proposed the normative model for decision under risk (i.e., expected utility theory). This theory is based on the assumption of the "independence axiom". However, compelling experimental data in behavioral economics showed that people's decision under risk violate such an assumption, which is modeled with nonlinear probability weighting functions in prospect theory [11]. The hyperbolic probability discounting model in behavioral psychology indicates that people over-devaluate likely (i.e., more immediate in repeated gambles) outcomes and under-devaluate unlikely (i.e., more delayed in repeated gambles) ones, which is in line with probability weighting function in prospect theory. Therefore, the hyperbolic probability discounting also represents the anomaly in decision under risk reported in behavioral economics, namely Allais' paradox.

We can now consider our possible common psychophysical accounts across intertemporal and probabilistic choices [2,3]. If time discounting and probability discounting share the common underlying mechanism of waiting time until the rewards, it is possible that anomalies in decision under risk may also be related to nonlinear psychophysical effect of subjective waiting time in (virtual) repeated gambles. Given that psychophysical time for delayed outcome is nonlinear, we may conjecture that psychological time for waiting an uncertain outcome (in repeated gambles) may also be in a logarithmic form:

$$
\tau(O)=\alpha \ln (1+\beta O)
$$

where $\alpha$ and $\beta$ are free parameters. Then we have a probability discount function with logarithmic waiting time for the uncertain outcome as

$$
V(O)=V(0) \cdot D(\tau(O))=\frac{V(0)}{(1+\beta O)^{k \alpha}}
$$

This formula can express hyperbola-like probability discount functions. Hence it is to be conjectured that by introducing nonlinear psychophysical time of uncertain rewards into the probability discounting models, we can also rationalize (i.e. "exponentialize") the functional form of probability discounting.

\subsection{The $q$-Exponential Discount Model for Decision over Time and under Risk}

In econophysics and behavioral biophysics, the $q$-exponential discount function based on Tsallis' non-extensive thermostatistics [12,13] was proposed to generalize the functional form of intertemporal choice [8,14-17]. The $q$-exponential time-discount function is:

$$
V(t)=\frac{V(0)}{[1+k(1-q) t]^{1 /(1-q)}}
$$

where $q$ is a free parameter. It can express both an exponential function $(q \rightarrow 1)$ and a hyperbolic function $(q=0)$. By considering the similarity between time and probability discounting behaviors, Takahashi (2007) [18] proposed the following $q$-exponential probability function:

$$
V(t)=\frac{V(0)}{[1+k(1-q) O]^{1 /(1-q)}}
$$

which has experimentally been verified [19].

The main objective of our current study is to empirically examine our theory that anomalies in decision over time and under risk can commonly be explained by nonlinearity of psychophysical time. If our theory is correct, the consideration of nonlinearity in subjective time for delayed outcome and uncertain outcome can rationalize ("exponentialize") functional forms of both time and probability discounting. We hypothesize that 1) both time discounting and probability discounting are in non-exponential forms in terms of physical time; 2) psychophysical time for real and virtual delays in waiting for delayed and uncertain rewards, respectively, are both in nonlinear forms; and 3) both time discounting and probability discounting are less deviated from the normative models (i.e. closer to exponential form) once psychophysical time is introduced into the models for time and probability discounting. 


\section{Method}

1) Participants: Thirty-three students $(M=24$, Mean age $=19.79$, $\mathrm{SD}=2.7$ ) from Hokkaido University participated in our study.

2) Procedure: We asked participants to perform paper-pencil tasks of time discounting and probability discounting (Takahashi, 2007). They were asked to indicate the choice between a hypothetical 100,000 yen reward available with seven delays (1 week, 2 weeks, 1 month, 6 month, 1 year, 5 years, 25 years) or seven probabilities $(95 \%, 90 \%, 70 \%, 50 \%, 30 \%, 10 \%, 5 \%)$ and a certain reward of various amounts (from 0 yen - 100,000 yen) available immediately. It is to be noted that the corresponding seven odds against values were calculated, by the experimenters (not by the participants) for data analysis with the definition of odds against $=1 /$ probability -1 , from the presented seven probabilities $(95 \%, 90 \%, 70 \%$, $50 \%, 30 \%, 10 \%, 5 \%$ ). In other words, odds against was not presented for participants.

For time perception tasks, they were asked to draw line on a $180 \mathrm{~mm}$ scale to indicate the length of psychological time until receiving delayed reward (e.g. "For how long do you feel you should wait until you receive the 100,000 yen 1 year later", corresponding to psychophysical time) and uncertain reward (e.g. "For how long do you feel you should wait until you win 100,000 yen with probability of $50 \%$ in repeated gambles", corresponding to subjective odds against).

Discounting data were analyzed as in our previous studies on temporal and probability discounting to determine the temporal and probability discount curves $[8$, $15,19,20]$. Briefly, we fist draw time and probability dis- count curves with physical (i.e., objective) time and odds against (i.e., $t$ and $O$ ), respectively. Next, we draw the time and probability discounting with subjective (psychophysical) time and odds against (i.e., $\tau(t)$ and $\tau(O)$ ), respectively. It is to be noted that this study is the first to measure subjective odds against (i.e., psychophysical waiting time in repeated gambles).

We used AIC (Akaike Information Criterion) to compare the fitness of the model, where the smaller values indicate the better fitness (tradeoff between overfitting and poor fitting).

\section{Result}

First, our study confirmed that both time discounting and probability discounting with physical time and objective odds against $(:=1 / p-1$, proportional to waiting time in virtual repeated gambles) were both in non-exponential forms, consistent with previous studies [19,21,22]. Namely, we fitted exponential, hyperbolic and $q$-exponential function to both discounting behaviors. The result of model fitness indicates that $q$-exponential function fitted the behavioral data best (for time discounting see Table $\mathbf{1}$ and Figure 1(a); probability discounting, Table 1 and Figure 2(a)). Furthermore, for both temporal and probability discount functions, parameter $q$ was closer to 0 than to 1 indicating both functional forms were "hyperbolic", rather than exponential (see Table 1).

Moreover, we also found that both psychophysical time for delayed outcome (subjective delay) and uncertain outcome (subjective odds against) were in a nonlinear form. Note again that waiting time in virtual repeated gambles in probability discounting is mentioned as "odds

Table 1. Parameters and AIC for time discounting and probability discounting of physical time and probability.

\begin{tabular}{cccccccc}
\hline & \multicolumn{3}{c}{ Time discounting } & \multicolumn{3}{c}{ Probability discounting } \\
\hline Model & Exponential $k$ & Hyperbolic $k$ & $k(q$-exp $)$ & $q(q$-exp $)$ & Exponential $k$ & Hyperbolic $k$ & $k(q$-exp $)$ \\
\hline Parameter & 0.011 & 0.03 & 19.9 & -3.5 & 3.6 & 9.1 & 717.3 \\
AIC & 148 & 147 & & 135 & 153 & -0.8 & 147 \\
\hline
\end{tabular}

The $q$-exponential model best fitted for both objective time and probability discounting. Time and probability discounting was hyperbolic rather than exponential, when physical time and odds against were utilized.

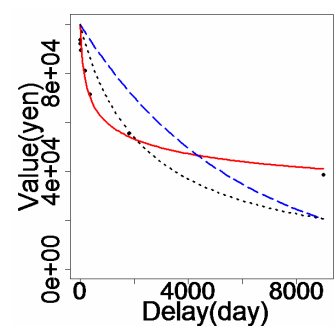

(a)

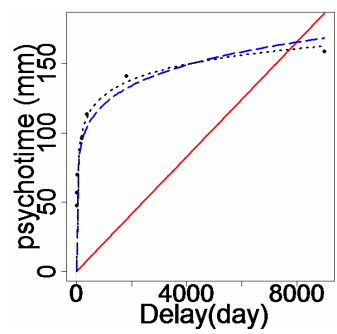

(b)

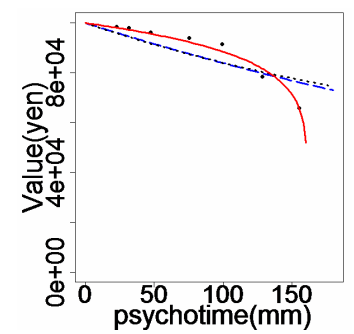

(c)

Figure 1. (a) Time discounting models with physical time; (b) Psychophysical time with physical time; (c) Time discounting with psychophysical time. For graph (a), (c), the red, blue dash and black dot curves are q-exponential, hyperbolic and exponential function respectively. For graph (b), the red, blue dash and black dot curves are linear, power and log function for psychophysical time (Y-axis) vs. physical time (delay, X-axis), respectively. 
against":= $1 / \mathrm{p}-1$ in behavioral psychology. We fitted linear $(V(t)=\alpha t)$ and power function $\left(V(t)=\alpha t^{\beta}\right)$ in addition to the log function to compare the fitness of the model. The results showed that the log function best fitted the data for both psychophysical time for delayed and uncertain rewards (for subjective time see Table 2 and Figure 1(b); for subjective odds against see Table 2 and Figure 2(b)), indicating that psychophysical time is logarithmic irrespective of the types of the decisions (i.e., decision over time and under risk). This study is the first to confirm that psychophysical time is commonly logarithmic across decisions under risk and over time.

Finally, we demonstrated that the functional form of time discounting and probability discounting were both closer to an exponential function (than simple hyperbolic function) after we introduced psychophysical time (Equation (4)) and subjective odds against (Equation (5)) for delayed and uncertain rewards respectively. The results of AIC again revealed that the $q$-exponential function fitted both types of discounting behaviors (see Table 3). In contrast to the discount functions with objective time and objective odds against, when psychophysical meas- urements were used to incorporate subjective time and psychophysical odds against, the parameter $q$ were closer to 1 rather than 0 for time discounting with subjective time and probability discounting with subjective odds against (see Table 3 and Figures 1(c) and 2(c)). This confirmed our theory in psychophysical neuroeconomics that nonlinear psychophysical time can rationalize (i.e. "exponentialize") both time discount and probability discount functions, indicating that anomalies in both intertemporal and probabilistic choices may result from nonlinearity of psychophysical time in decision over time (subjective delay) and under risk (subjective odds against).

\section{Discussion}

The common time-perception account of anomalies in decision over time and under risk in psychophysical neuroeconomics $[2,3,7,9,16]$ has, for the first time, been confirmed in the present experimental study. Our current study has adopted the perspective of psychophysical law of time perception to account for those anomalies. Therefore, it can be speculated that common neural informa-

Table 2. Parameters and AIC of psychophysical time for delayed rewards and uncertain rewards.

\begin{tabular}{|c|c|c|c|c|c|c|c|c|c|c|}
\hline \multirow{3}{*}{ Model } & \multicolumn{5}{|c|}{ Psychophysical time for delayed reward } & \multicolumn{5}{|c|}{ Psychophysical time for uncertain reward } \\
\hline & \multirow{2}{*}{$\begin{array}{c}\text { Linear } \\
\alpha\end{array}$} & \multicolumn{2}{|c|}{ Power } & \multicolumn{2}{|c|}{ Logarithmic } & \multirow{2}{*}{$\begin{array}{c}\text { Linear } \\
A\end{array}$} & \multicolumn{2}{|c|}{ Power } & \multicolumn{2}{|c|}{ Logarithmic } \\
\hline & & $\alpha$ & $\beta$ & $\alpha$ & $\beta$ & & $\alpha$ & $\beta$ & $\alpha$ & $\beta$ \\
\hline Parameter & 0.02 & 58.9 & 0.18 & 23.9 & $3.4 \times 10^{13}$ & 10.1 & 66.3 & 0.34 & 52.9 & 7047.4 \\
\hline AIC & 84.4 & \multicolumn{2}{|c|}{63.4} & \multicolumn{2}{|c|}{59.4} & 75.9 & \multicolumn{2}{|c|}{60.5} & \multicolumn{2}{|c|}{55.8} \\
\hline
\end{tabular}

Weber-Fechner law best fitted for psychophysical time and odds against.

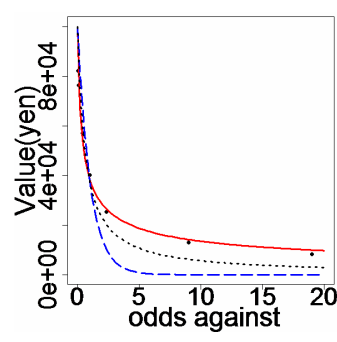

(a)

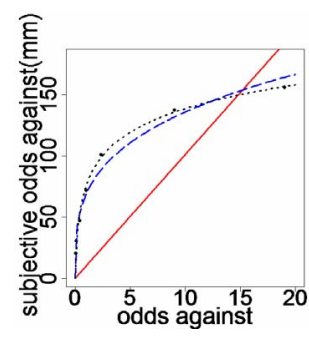

(b)

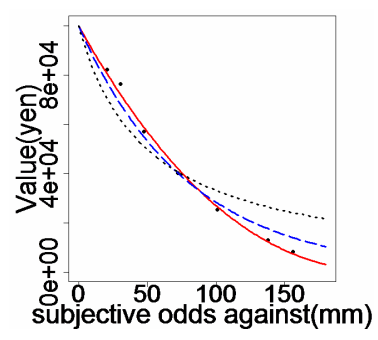

(c)

Figure 2. (a) Probability discounting with objective odds against (waiting time); (b) Psychophysical odds against with objective odds against; (c) Probability discounting with psychophysical odds against. For graph (a), (c), the red, blue dash and black dot curves are q-exponential, hyperbolic and exponential function respectively. For graph (b), the red, blue dash and black dot curves are linear, power and log functions for psychophysical odds against; i.e., subjective waiting time in repeated gambles (Y-axis) and physical waiting time in repeated gambles, respectively.

Table 3. Parameters and AIC for time discounting and probability discounting with subjective time for delayed and uncertain rewards.

\begin{tabular}{ccccccccc}
\hline & \multicolumn{3}{c}{ Time discounting (subjective time) } & \multicolumn{4}{c}{ Probability discounting (subjective time) } \\
\hline Model & Exponential $k$ & Hyperbolic $k$ & $k(q$-exp) & $q(q$-exp) & Exponential $k$ & Hyperbolic $k$ & $k(q$-exp) & $q(q$-exp) \\
\hline Parameter & 0.0051 & 0.012 & 0.00027 & 28.4 & 0.12 & 0.21 & 0.074 & 1.01 \\
AIC & 153 & 155 & \multicolumn{1}{c}{140} & 155 & 156 & 150 \\
\hline
\end{tabular}

The $q$-exponential model best fitted for both objective time and probability discounting. Time and probability discounting was exponential rather than hyperbolic, when psychophysical (subjective) time and odds against were utilized. 
tion processing may cause the anomalies in intertemporal choice and under risk, which should be investigated in future studies in behavioral neuroeconomics. Furthermore, studies in quantum decision theory [23-29] which utilize quantum probability theory for modeling human probability judgment and probabilistic choice, should consider the psychophysical equivalence of time and probability, which was confirmed in the current study. Also, cultural differences in these psychophysical processing should be investigated in order to resolve the cause of cultural differences in economic decision making and perception of economic growth [30,31].

\section{REFERENCES}

[1] D. Prelec and G. Loewenstein, "Decision-Making over Time and under Uncertainty: A Common Approach," Management Science, Vol. 37, No. 7, 1991, pp. 770-786. http://dx.doi.org/10.1287/mnsc.37.7.770

[2] T. Takahashi, "Psychophysics of the Probability Weighting Function," Physica A: Statistical Mechanics and its Applications, Vol. 390, No. 5, 2011, pp. 902-905.

[3] T. Takahashi, R. Han and F. Nakamura, "Time Discounting: Psychophysics of Intertemporal and Probabilistic Choices," Journal of Behavioral Economics and Finance, Vol. 5, 2012, pp. 10-14.

[4] H. Rachlin, A. Raineri and D. Cross, "Subjective Probability and Delay," Journal of Experimental Analysis of Behavior, Vol. 55, No. 2, 1991, pp. 233-244. http://dx.doi.org/10.1901/jeab.1991.55-233

[5] P. A. Samuelson, "A Note on Measurement of Utility," The Review of Economic Studies, Vol. 4, No. 2, 1937, pp. 155-161. http://dx.doi.org/10.2307/2967612

[6] R. H. Strotz, "Myopia and Inconsistency in Dynamic Utility Maximizatio," Review of Economic Studies, Vol. 23, No. 3, 1955, pp. 165-180. http://dx.doi.org/10.2307/2295722

[7] T. Takahashi, "Loss of Self-Control in Intertemporal Choice May Be Attributable to Logarithmic Time-Perception," Medical Hypotheses, Vol. 65, No. 4, 2005, pp. 691-693. http://dx.doi.org/10.1016/j.mehy.2005.04.040

[8] R. Han and T. Takahashi, "Psychophysics of Valuation and Time Perception in Temporal Discounting of Gain and Loss," Physica A: Statistical Mechanics and Its Applications, Vol. 391, No. 24, 2012, pp. 6568-6576. http://dx.doi.org/10.1016/j.physa.2012.07.012

[9] T. Takahashi, "A Neuroeconomic Theory of Rational Addiction and Nonlinear Time-Perception," Neuro Endocrinology Letters, Vol. 32, No. 3, 2011, pp. 221-225.

[10] J. von Neumann and O. Morgenstern, "Theory of Games and Economic Behavior," Princeton University Press, Princeton, 1947.

[11] D. Kahneman and A. Tversky, "Prospect Theory: An Analysis of Decision under Risk," Econometrica, Vol. 47, No. 2, 1979, pp. 263-292. http://dx.doi.org/10.2307/1914185

[12] Tsallis, C. Anteneodo, L. Borland and R. Osorio, "Non- extensive Statistical Mechanics and Economics," Physica A: Statistical Mechanics and its Applications, Vol. 324, No. 1-2, 2003, pp. 89-100.

[13] C. Tsallis, "Nonadditive Entropy Sq and Nonextensive Statistical Mechanics: Applications in Geophysics and Elsewhere," ActaGeophysica, Vol. 60, No. 3, 2012, pp. 502-525. http://dx.doi.org/10.2478/s11600-012-0005-0

[14] D. O. Cajueiro, "A Note on the Relevance of the $q$-Exponential Function in the Context of Intertemporal Choices," Physica A: Statistical Mechanics and Its Applications, Vol. 364, 2006, pp. 385-388. http://dx.doi.org/10.1016/j.physa.2005.08.056

[15] T. Takahashi, H. Oono and M. H. B. Radford, "Psychophysics of Time Perception and Intertemporal Choice Models," Physica A: Statistical Mechanics and Its Applications, Vol. 387, No. 8-9, 2008, pp. 2066-2074. http://dx.doi.org/10.1016/j.physa.2007.11.047

[16] T. Takahashi, "Theoretical Frameworks for Neuro-Economics of Intertemporal Choice," Journal of Neuroscience, Psychology, and Economics, Vol. 2, No. 2, 2009, pp. 75-90. http://dx.doi.org/10.1037/a0015463

[17] T. Takahashi, "Molecular Neuroeconomics of Crime and Punishment: Implications for Neurolaw," NeuroEndocrinology Letters, Vol. 33, No. 7, 2012, pp. 667-673.

[18] T. Takahashi, "A Probabilistic Choice Model Based on Tsallis' Statistics," Physica A: Statistical Mechanics and its Applications, Vol. 386, No. 1, 2007, pp. 335-338.

[19] T. Takahashi, R. Han, H. Nishinaka, T. Makino and H. Fukui, "The $q$-Exponential Probability Discounting of Gain and Loss," Applied Mathematics, Vol. 4, No. 6, 2013, pp. 876-881. http://dx.doi.org/10.4236/am.2013.46120

[20] T. Takahashi, H. Oono and M. H. B. Radford, "Empirical Estimation of Consistency Parameter in Intertemporal Choice Based on Tsallis' Statistics," Physica A: Statistical Mechanics and Its Applications, Vol. 381, 2007, pp. 338-342. http://dx.doi.org/10.1016/j.physa.2007.03.038

[21] T. Takahashi, K. Ikeda and T. Hasegawa, "A Hyperbolic Decay of Subjective Probability of Obtaining Delayed Rewards," Behavior and Brain Functions, Vol. 3, 2007, p. 52. http://dx.doi.org/10.1186/1744-9081-3-52

[22] T. Takahashi, H. Oono and M. H. Radford, "Comparison of Probabilistic Choice Models in Humans," Behavioral and Brain Functions, Vol. 3, No. 1, 2007, p. 20.

http://dx.doi.org/10.1186/1744-9081-3-20

[23] M. Asano, I. Basieva, A. Khrennikov, M. Ohya and I. Yamato, "Non-Kolmogorovian Approach to the ContextDependent Systems Breaking the Classical Probability Law," Foundations of Physics, Vol. 43, No. 7, 2013, pp. 895-911. http://dx.doi.org/10.1007/s10701-013-9725-5

[24] J. R. Busemeyer, E. Pothos, R. Franco and J. S. Trueblood, "A Quantum Theoretical Explanation for Probability, Judgment 'Errors'," Psychological Review, Vol. 118, No. 2, 2011, pp. 193-218. http://dx.doi.org/10.1037/a0022542

[25] T. Cheon and T. Takahashi, "Interference and Inequality in Quantum Decision Theory," Physics Letters A, Vol. 375, No. 2, 2010, pp. 100-104. 
http://dx.doi.org/10.1016/j.physleta.2010.10.063

[26] T. Cheon and T. Takahashi, "Quantum Phenomenology of Conjunction Fallacy," Journal of the Physical Society of Japan, Vol. 81, No. 10, 2012, Article ID: 104801. http://dx.doi.org/10.1143/JPSJ.81.104801

[27] T. Takahashi, "Quantum Decision Theory for Computational Psychiatry," NeuroQuantology, Vol. 10, No. 4, 2012, pp. 688-691.

[28] A. Y. Khrennikov, "Ubiquitous Quantum Structure: From Psychology to Finance," Springer-Verlag, Berlin, 2010. http://dx.doi.org/10.1007/978-3-642-05101-2
[29] V. I. Yukalov and D. Sornette, "Decision Theory with Prospect Interference and Entanglement," Theory and Decision, Vol. 70, No. 3, 2011, pp. 283-328. http://dx.doi.org/10.1007/s11238-010-9202-y

[30] T. Takahashi, T. Hadzibeganovic, S. A. Cannas, T. Makino, H. Fukui and S. Kitayama, "Cultural Neuroeconomics of Intertemporal Choice," NeuroEndocrinology Letters, Vol. 30, No. 2, 2009, pp. 185-191.

[31] W. A. Wagenaar and S. Sagaria, "Misperception of Exponential Growth," Perception and Psychophysics, Vol. 18 , No. 6, 1975, pp. 416-422. http://dx.doi.org/10.3758/BF03204114 